\title{
New simple form for phenomenological nuclear potential
}

\author{
P. Salamon, T. Vertse \\ Institute of Nuclear Research of the Hungarian \\ Academy of Sciences, H-4001 Debrecen, P. O. Box 51, \\ University of Debrecen, Faculty of Informatics, \\ H-4010 Debrecen, P. O. Box 12, Hungary
}

(Dated: November 20, 2009)

\begin{abstract}
A simple form is introduced for phenomenological nuclear potential as an alternative of the popular Woods-Saxon (WS) form. In contrast to the WS form the new form becomes exactly zero at a finite distance and all derivatives of this form is continuous everywhere. The sequence of the single particle levels of a WS potential with realistic parameters can be reproduced reasonably well with the new potential in which even the positions of the broad resonances are free from uncertainties.
\end{abstract}

PACS numbers: 21.10.Pc,21.10.Ma,21.30.-x,21.60.Cs,23.90.+w 
Considerable interest has been focused recently to the resonant states because the radioactive nuclear beam facilities produce slightly bound and unbound nuclei. New theoretical approach has been developed for an efficient description of these nuclei in terms of unbound single particle levels. The shell model has been generalized for the description of the unbound levels using complex energy resonant states (Gamow states) in the shell model basis[1]-[2]. The single particle potential used in these calculations has a Woods-Saxon form since self-consistent calculations show that the mean nuclear field felt by a single nucleon can be approximated reasonably well by a phenomenological Woods-Saxon form:

$$
V^{W S}(r)=-\frac{V_{0}}{1+e^{\frac{r-R}{a}}},
$$

complemented by a spin-orbit term having a derivative WS shape:

$$
V_{s o}^{W S}(r)=-\frac{V_{s o}}{r a} 2(\vec{l} \cdot \vec{s}) \frac{e^{\frac{r-R}{a}}}{\left(1+e^{\frac{r-R}{a}}\right)^{2}} .
$$

Due to the simplicity of the WS shape this form is used almost exclusively not only in nuclear structure calculations but for the description of scattering.

The WS form however has an infinite range which is in contradiction with the short range property of the nuclear forces. In order to cure this false property a truncated version is used in which the WS shape is cut to zero at a certain finite $R_{\max }$ distance, where the magnitude of the potential is reasonably small. In practical calculations only the truncated WS form

$$
V_{t r}^{W S}(r)=\left\{\begin{aligned}
V^{W S}(r), & \text { if } r<R_{\max } \\
0, & \text { if } r \geq R_{\max }
\end{aligned}\right.
$$

is used. (Due to the sharp truncation $\frac{d V_{t r}^{W S}}{d r}(r)$ does not exists at $r=R_{\max }$.) The same truncation is introduced for the spin-orbit term $\left(V_{s o}^{t r}(r)\right)$, therefore the phenomenological nuclear potential is zero beyond $R_{\max }$. If we chose a matching radius $R_{m} \geq R_{\max }$ we can match the solutions of the radial Schrödinger equation with the truncated WS potential to the asymptotic solution (spherical Bessel and Hankel functions) and we can calculate a number of poles for the $S$-matrix on the complex energy $(E)$ or wave number $(k)$ planes. Physical resonances belong to poles of the $S$-matrix lying close to the real axes. In a given partial wave we have at most one narrow resonance and a number of broad ones. It is well known that the position of the narrow resonances depends weakly on the truncation radius $R_{\text {max }}[3]$. However the distribution of the broad resonant poles depends strongly on the value 


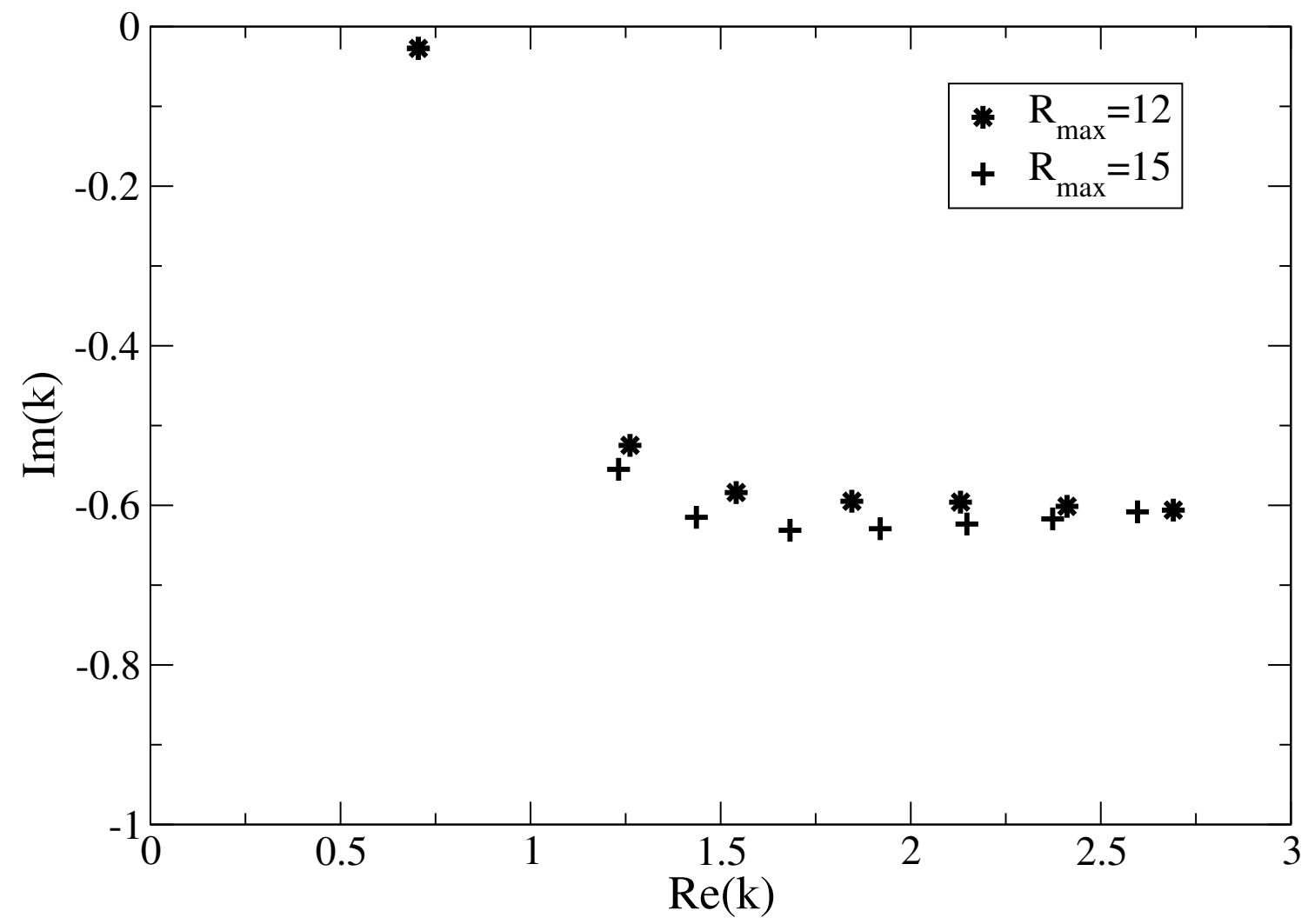

FIG. 1: Positions of the $1 h_{11 / 2}$ resonance poles on the complex $k$-plane calculated for a truncated Woods-Saxon potential with $V_{0}=50 \mathrm{MeV}, R=4.6 \mathrm{fm}, a=0.65 \mathrm{fm}$ with $R_{\max }=15 \mathrm{fm}$ (plus) and with $R_{\max }=12 \mathrm{fm}$ (star), respectively.

of $R_{\max }$, and this represent another inconvenient feature of the truncated WS potential. In Fig. 1 we show examples for this dependence. While the position of the physical $1 h_{11 / 2}$ resonance at $E=(10.49,-0.81) \mathrm{MeV}, k=(0.705,-0.027) \mathrm{fm}^{-1}$ is practically independent of the value of $R_{\max }$ used, the positions of the other resonances strongly depend on the $R_{\max }$ value. These broad resonances proved to be very useful in the pole expansion of the Green-function and the response function in an approximation to the continuum RPA [4].

The origin of the $R_{\max }$ dependence can be explained if we calculate the energy shift of a pole perturbatively. Let us denote the energy of the $n$-th pole and the corresponding normalized radial wave function by $\epsilon_{n}$ and $u_{i}\left(r, k_{n}\right)$, respectively for the potential $V_{t r}^{W S}$ with truncation radius $R_{\max }$. (For the normalization of the pole solutions we used the generalized scalar product of Berggren [5] and the complex rotation introduced in Ref. [6].) Let us 
increase the truncation radius $R_{\max }$ by $\Delta R$. The corresponding change of the pole energy is

$$
\Delta \epsilon_{n}=\int_{R_{\max }}^{R_{\max }+\Delta R} V^{W S}(r) u_{i}^{2}\left(r, k_{n}\right) d r \neq 0 .
$$

Since the WS potential is not zero in the region of the integration, the energy change $\Delta \epsilon_{n}$ depends on the behavior of the radial wave function $u_{i}\left(r, k_{n}\right)$ in the integration region. For well bound states and for narrow resonances $u_{i}\left(r, k_{n}\right)$ is small in that region and the energy shift is negligible. For broad resonances, however, the radial wave functions could be large in the integration region and this might result large $\Delta \epsilon_{n}$ energy shifts (see Fig. 1).

Since in the majority of the publications the $R_{\max }$ values are not specified therefore the position of the broad resonances might be quite uncertain.

Our aim in this Brief Report is to find a potential which becomes zero exactly at a finite $\rho$ distance and has continuous derivatives everywhere (even at $r=\rho$ ). For such a potential the pole energy will evidently not depend on the matching radius $R_{m}$ if the matching radius $R_{m}>\rho$.

A good candidate for the finite range form is the following:

$$
f_{\rho}(r)=\left\{\begin{aligned}
-e^{\frac{r^{2}}{r^{2}-\rho^{2}}}, & \text { if } r<\rho \\
0, & \text { if } r \geq \rho
\end{aligned}\right.
$$

since

$$
\lim _{r \rightarrow \rho} f_{\rho}(r)=0 .
$$

A nice feature of this form is that its derivatives of all orders disappear at $r=\rho$.

$$
\left.\frac{d^{n} f_{\rho}(r)}{d r^{n}}\right|_{r=\rho}=0 \quad n=1,2, \ldots
$$

Therefore the derivatives are continuous even in the $r=\rho$ point.

A simple potential form of this type:

$$
V(r)=c_{0} f_{\rho}(r)
$$

is considerably different from the WS shape. Since the truncated WS potential in Eq.(3) has four parameters: $V_{0}, R, a$ and $R_{\max }$ we try to approximate it by a new finite range form which also has four free parameters. The shape of the new potential has to be improved in the surface region, therefore we add a term which has its maximum at the surface. The first derivative of the previous form 


$$
f_{\rho}^{\prime}(r)=\frac{2 r \rho^{2}}{\left(r^{2}-\rho^{2}\right)^{2}} e^{\frac{r^{2}}{r^{2}-\rho^{2}}}
$$

has this behavior. Therefore our new potential will be a combination of these terms with adjustable strengths $c_{0}$ and $c_{1}$ and radii $\rho_{0}, \rho_{1}$ :

$$
V(r)=c_{0} f_{\rho_{0}}(r)+c_{1} f_{\rho_{1}}^{\prime}(r) \equiv V\left(r, c_{0}, c_{1}, \rho_{0}, \rho_{1}\right) .
$$

In order to get the best fit of the new potential to the central WS term we minimize the integral

$$
\Delta\left(\rho_{0}, \rho_{1}, c_{0}, c_{1}\right)=\int_{0}^{\rho_{>}}\left[V\left(r, c_{0}, c_{1}, \rho_{0}, \rho_{1}\right)-V_{t r}^{W S}(r)\right]^{2} d r,
$$

as function of the new parameters, where $\rho_{>}=\max \left\{\rho_{0}, \rho_{1}\right\}$. The parameters of the WS potential are kept fixed during the minimization procedure. We try to reproduce the WS potential of the $A=50$ nucleus with typical parameters: $V_{0}=50 \mathrm{MeV}, r_{0}=1.25 \mathrm{fm}, a=0.65$ $\mathrm{fm}$ and $R_{\max }=15 \mathrm{fm}$. In order to find the absolute minimum of the multi-variable function $\Delta$ we used first the downhill simplex method [7] with several starting simplexes chosen randomly. Later we turned to the use of Powell's method [8] for finding the position of the minimum of the integral in Eq.(11). The best fit value $\Delta_{\min }=6.8$ belongs to the parameters: $\rho_{0}=7.1 \mathrm{fm}, \rho_{1}=4.78 \mathrm{fm}, c_{0}=49.8 \mathrm{MeV}$ and $c_{1}=-14.59 \mathrm{MeV}$.

One can see in Fig. 2 that the new finite range potential form in Eq.(10) with these parameters reproduces the general features of the central WS shape reasonably well. The largest difference occurs between the two potential forms in the external region. Here the new potential becomes zero at $\rho_{0}=7.1 \mathrm{fm}$, while the truncated WS term cut to zero only at $15 \mathrm{fm}$. We try to find some relation between the parameters of the new and the WS potentials. In this case $c_{0} \approx V_{0}$ and the radius $\rho_{1}=4.78 \mathrm{fm}$ is a little larger than the radius of the WS well $1.25 A^{1 / 3}=4.6 \mathrm{fm}$.

In order to check if these relations are typical we varied the mass number $A$ in the radius of the Woods-Saxon potential: $R=1.25 A^{1 / 3}$ and calculated the best fit values of the parameters of the new potential. We noticed that indeed $c_{0} \approx V_{0}$ and $\rho_{1} / A^{1 / 3}$ was almost constant with a value which is a bit larger than $r_{0}$. Therefore $\rho_{1}$ resembles to the $R$ radius of the WS potential. The difference of the radii plays the same role as the diffuseness of the WS form, since

$$
\rho_{0}-\rho_{1} \approx 4 a
$$




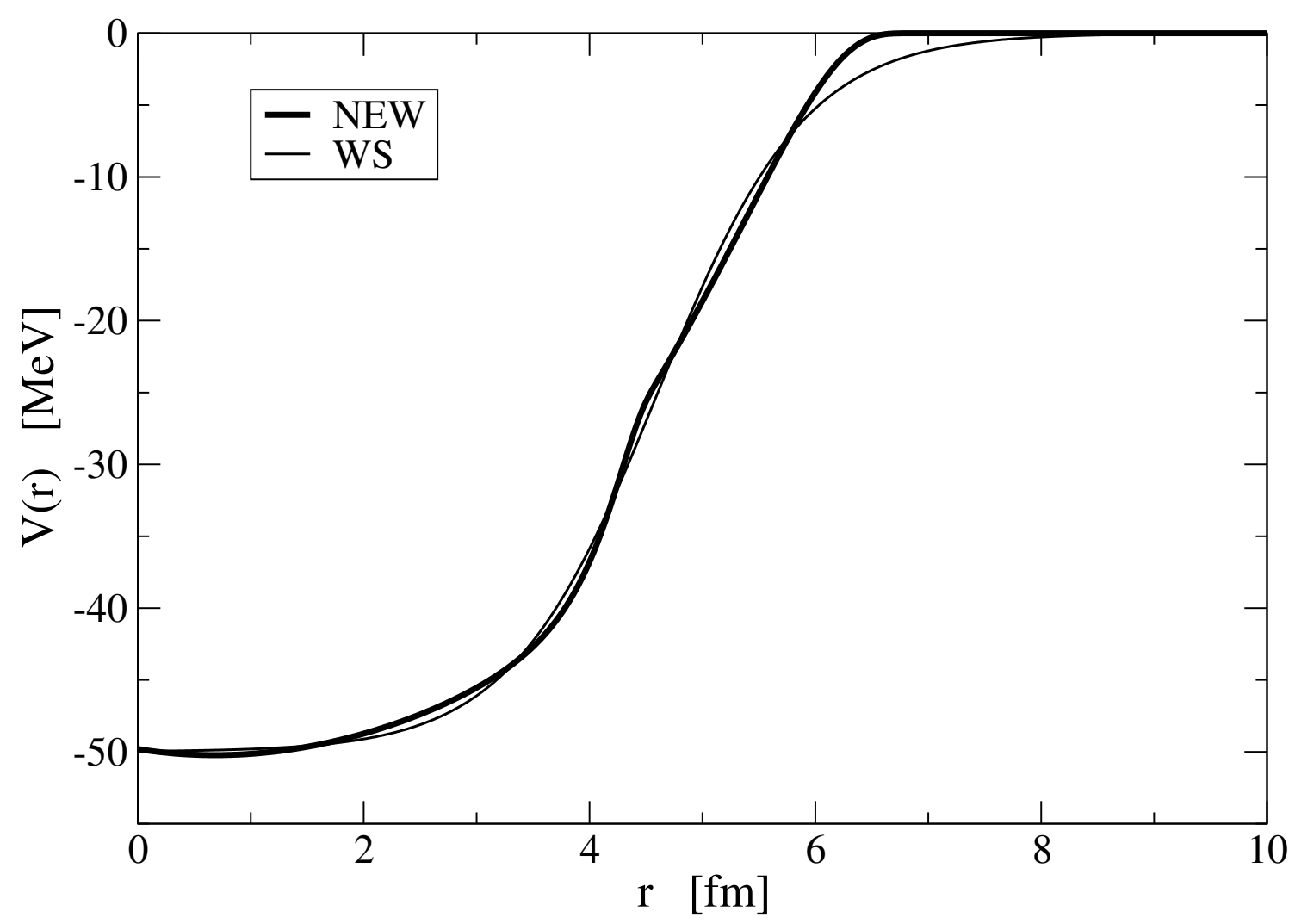

FIG. 2: Comparison of the central part of the Woods-Saxon potential with $V_{0}=50 \mathrm{MeV}, \quad R=4.6$ $\mathrm{fm}, a=0.65 \mathrm{fm}$ with the new potential with parameters $\rho_{0}=7.1 \mathrm{fm}, \rho_{1}=4.78 \mathrm{fm}, c_{0}=49.8 \mathrm{MeV}$ and $c_{1}=-14.59 \mathrm{MeV}$.

with the exception of very light nuclei $A<28$.

An important comparison of the two phenomenological potentials can be done if we calculate the single particle spectra they produce.

In order to have a reasonable shell structure we have to fix the strength and the shape of the spin-orbit potential. We calculated the bound and resonant single-particle energies using this spin-orbit strength $V_{s o}=10 \mathrm{MeV}$ in Eq.(2). The single particle energy values were calculated by using the computer code GAMOW [9]. The values we received for the WS potential are shown in the second column of Table I.

For the new finite range potential we used a spin-orbit form which is $1 / r$ times the derivative of the central potential of Eq.(10): 
TABLE I: Energies of the single-particle states corresponding to the WS and the new finite range potentials in Eqs.(10) and (13) for neutrons outside the $A=50$ core. Energies are in $\mathrm{MeV}$.

\begin{tabular}{lcc}
\hline State & Energy(WS $)$ & Energy(New) \\
\hline $1 s_{1 / 2}$ & -39.14 & -38.96 \\
$1 p_{3 / 2}$ & -30.15 & -30.13 \\
$1 p_{1 / 2}$ & -28.93 & -28.50 \\
$1 d_{5 / 2}$ & -20.26 & -20.53 \\
$1 d_{3 / 2}$ & -17.67 & -17.16 \\
$2 s_{1 / 2}$ & -17.15 & -17.29 \\
$1 f_{7 / 2}$ & -9.81 & -10.42 \\
$1 f_{5 / 2}$ & -5.72 & -5.05 \\
$2 p_{3 / 2}$ & -6.78 & -6.78 \\
$2 p_{1 / 2}$ & -5.50 & -5.03 \\
$2 d_{5 / 2}$ & $(1.05,-0.23)$ & $(1.29,-0.29)$ \\
$2 d_{3 / 2}$ & $(1.93,-0.92)$ & $(2.85,-1.61)$ \\
$1 g_{9 / 2}\left(0.72,-8.8 \times 10^{-3}\right)$ & $(-0.10,0.0)$ \\
$1 g_{7 / 2}$ & $(5.58,-0.30)$ & $(6.58,-0.40)$ \\
\hline
\end{tabular}

$$
\begin{aligned}
V_{\text {so }}^{\text {new }}(r) & =-\frac{c_{s o}}{r c_{0}} 2(\vec{l} \cdot \vec{s}) \frac{d V(r)}{d r}= \\
& =-\frac{c_{s o}}{r} 2(\vec{l} \cdot \vec{s})\left[f_{\rho_{0}}^{\prime}(r)+\frac{c_{1}}{c_{0}} f_{\rho_{1}}(r) \frac{2 \rho_{1}^{2}\left(3 r^{4}-\rho_{1}^{4}\right)}{\left(r^{2}-\rho_{1}^{2}\right)^{4}}\right]
\end{aligned}
$$

In the third column of Table I we present the bound and resonant state energies calculated by using the new central potential in Eq.(10) and the new spin-orbit term in Eq.(13) with $c_{s o}=0.267 c_{0}$. This strength of the new spin-orbit term belongs to the minimum of the sum of squared differences between the bound state energies listed in the second and the third columns in Table I.

One can see that the overall shell structure of the spectrum produced by the WS potential is reproduced reasonably well by the new potential. The largest differences are for the $1 f_{5 / 2}$ $(0.67 \mathrm{MeV})$ and $1 f_{7 / 2}(0.61 \mathrm{MeV})$ bound orbits. For the resonant orbits the differences do not exceed $1 \mathrm{MeV}$. 


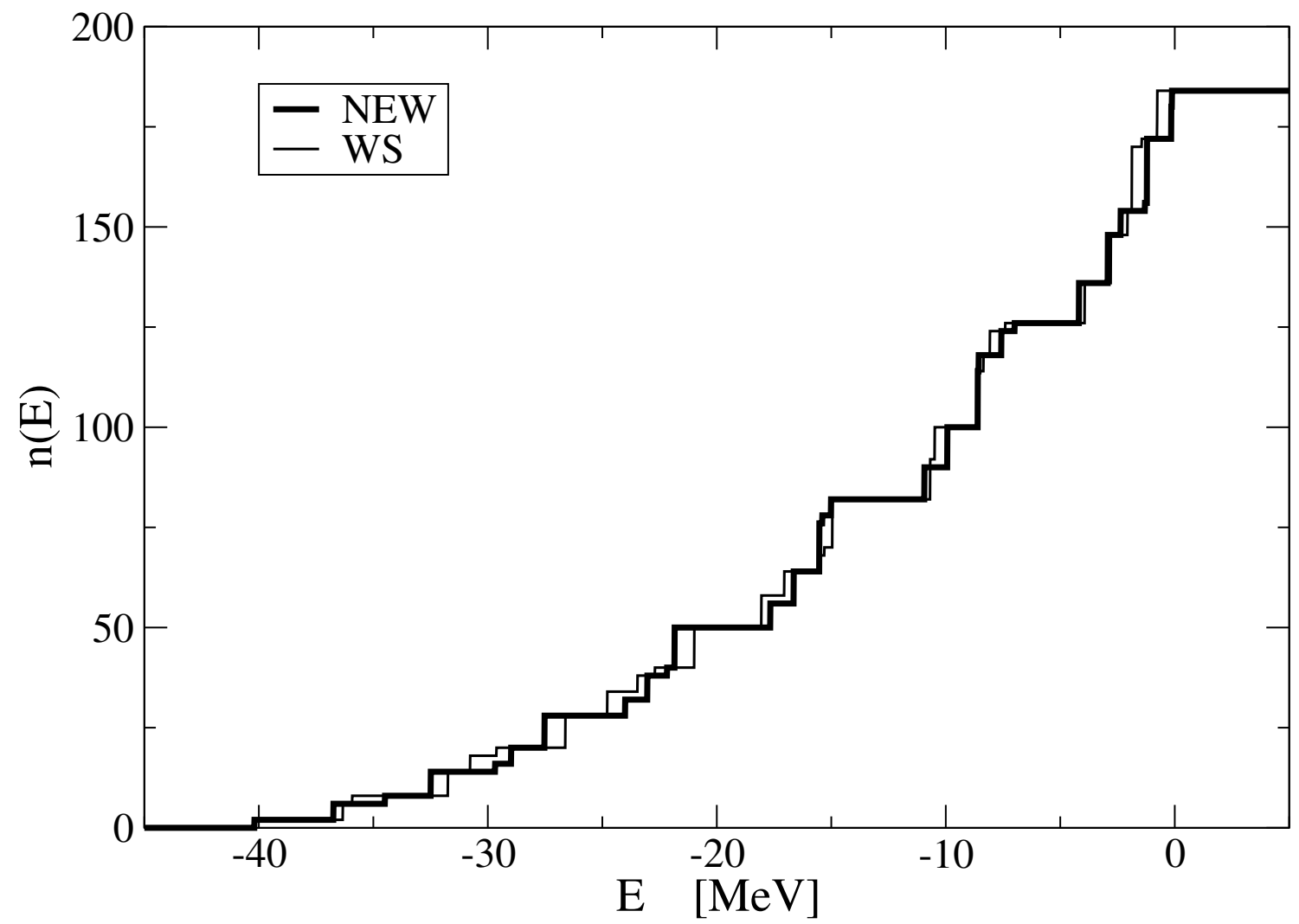

FIG. 3: Comparison of the particle numbers in Eq.(14) calculated for neutrons outside the $A=208$ core for the new potential in Eq.(10) with parameters $\rho_{0}=10.96 \mathrm{fm}, \rho_{1}=8.33 \mathrm{fm}, c_{0}=43.66 \mathrm{MeV}$ and $c_{1}=-44.27 \mathrm{MeV}$ and spin-orbit strengths $c_{s o}=0.38 c_{0}$ to the particle number of the Woods-Saxon potential with parameters in [10].

We have to check if the spectrum of the WS potential can be reproduced well by the new potential form for heavy nuclei. Therefore we calculated single particle neutron states outside ${ }^{208} \mathrm{~Pb}$ core. The parameters of the truncated WS potential have been taken from Ref. [10] $\left(V_{0}=44.4 \mathrm{MeV}, r_{0}=1.27 \mathrm{fm}, a=0.7 \mathrm{fm}, R_{\max }=15 \mathrm{fm}, V_{\text {so }}=16.5 \mathrm{MeV}\right)$. This WS potential was fitted with the new finite range form in Eq.(10). The central potential was reproduced reasonably well and the strength of the new spin-orbit term $c_{s o}=0.38 c_{0}$ gave the best fit to all bound state energies with the WS case.

The single particle energies (lying above the Fermi level) calculated with the WS and the new potentials are compared in Table II. The particle numbers

$$
n(E)=\sum_{i: \epsilon_{i}<E}\left(2 j_{i}+1\right),
$$

with the bound state energies calculated for the new and for the WS potentials are displayed 
TABLE II: Energies of the single-particle states lying above the Fermi level corresponding to the WS and the new finite range potentials in Eqs.(10) and (13) for neutrons outside the $A=208$ core. Energies are in $\mathrm{MeV}$.

\begin{tabular}{lcc}
\hline State & Energy $(\mathrm{WS})$ & Energy(New) \\
\hline $2 g_{9 / 2}$ & -3.93 & -3.92 \\
$1 i_{11 / 2}$ & -2.80 & -2.81 \\
$3 d_{5 / 2}$ & -2.07 & -2.00 \\
$1 j_{15 / 2}$ & -1.88 & -1.97 \\
$4 s_{1 / 2}$ & -1.44 & -1.31 \\
$3 d_{3 / 2}$ & -0.78 & -0.63 \\
$2 g_{7 / 2}$ & -0.77 & -0.50 \\
$3 f_{7 / 2}$ & $(2.10,-0.87)$ & $(2.33,-0.95)$ \\
$2 h_{11 / 2}$ & $(2.25,-0.026)$ & $\left(2.41,-3.1 \times 10^{-2}\right)$ \\
$3 f_{5 / 2}$ & $(2.70,-2.32)$ & $(3.45,-2.59)$ \\
$1 k_{17 / 2}$ & $\left(5.03,-1.26 \times 10^{-3}\right)$ & $\left(4.87,-9 \times 10^{-4}\right)$ \\
$1 j_{13 / 2}$ & $\left(5.41,-9.4 \times 10^{-3}\right)$ & $\left(5.36,-8 \times 10^{-3}\right)$ \\
\hline
\end{tabular}

in Fig. 3. One can see that the single particle energies calculated by using the WS and the finite range potentials agree well.

In summary in this Brief Report we introduced a new phenomenological form for the nuclear potential which has continuous derivatives everywhere and has a finite range. We showed that a typical WS form can be approximated reasonably well with the new form in Eq.(10). From the new parameters $c_{0}$ resembles to $V_{0}$ and $\rho_{1}$ resembles to $R$. The range $\rho_{0}$ of the new potential takes over the role of the cutoff radius of the WS potential, although its value is much smaller than $R_{\max }$. The difference $\rho_{0}-\rho_{1}$ correlates with the $a$ diffuseness of the WS form. If we complement the new potential with a spin-orbit term in Eq.(13) the single particle spectra of the WS and the new forms are very similar even in the resonant region. For a matching distance $R_{m} \geq \rho_{0}$ the pole position is independent of $R_{m}$. If we use the new form the range of the nuclear interaction is defined unambiguously in contrast to the WS form. 
Therefore we strongly suggest to use the form in Eq.(10) as a new finite range potential instead of the truncated Woods-Saxon form. A systematic study for determining the equivalent finite range potential parameters to the most frequently used potential families with WS form is in progress.

Acknowledgement: Authors thank to A. T. Kruppa for valuable discussions and for the Hungarian Research Fund OTKA (No. T46791) for the support.

[1] R. Id Betan, R. J. Liotta, N. Sandulescu, T. Vertse, Phys. Rev. Lett. 89, 042501 (2002).

[2] N. Michel,W. Nazarewicz, M. Ploszajczak, K. Bennaceur, Phys. Rev. Lett. 89, 042502 (2002).

[3] A. I. Baz', Ya. B. Zeldovich, and A. M. Perelomov, Scattering, Reactions, and Decay in Non-relativistic Quantum Mechanics, IPST No. 5149 Jerusalem, (1969).

[4] P. Lind, R. J. Liotta, E. Maglione, T. Vertse, Z. Phys. A 347, 231 (1994).

[5] T. Berggren, Nucl. Phys. A109, 265 (1968).

[6] B. Gyarmati, T. Vertse, Nucl. Phys, A160, 523 (1971).

[7] J. A. Nelder, R. Mead, Comput. J., 7, 308 (1965).

[8] W. H. Press, S. A. Theukolsky, W. T. Vetterling, B. P. Flannery, Numerical Recipes in FORTRAN, The Art of Scientific Computing, Second Edition, (Cambridge University Press, Cambridge 1992).

[9] T. Vertse, K. F. Pál, Z. Balogh, Comput. Phys. Commun. 27, 309 (1982).

[10] P. Curutchet, T. Vertse, and R. J. Liotta, Phys. Rev. C39, 1020 (1989). 